Pure Appl. Chem., Vol. 73, No. 3, pp. 613-616, 2001.

(C) 2001 IUPAC

\title{
Molecules and macromolecules involved in chemical communication of scarab beetles*
}

\author{
Walter Soares Leal \\ Department of Entomology, University of California, Davis, CA 95616 USA
}

\begin{abstract}
Chemical communication involves the production and release of specific chemicals (pheromones and other semiochemicals) by the emitter, and the detection and olfactory processing of these signals leading to appropriate behavioral responses in the receiver. In contrast to most of the scarab species investigated to date, the Japanese and Osaka beetles have the ability to detect the allospecific pheromone, which plays a pivotal role in the isolation mechanism between these two species. Each species produces a single enantiomer of japonilure [(Z)-5-(dec1-enyl)oxacyclopentan-2-one], but they have evolved the ability to detect both enantiomers, one as an attractant and the other as a behavioral antagonist (stop signal). There is growing evidence in the literature that the inordinate sensitivity and selectivity of the insect olfactory system is achieved by a combination of various olfactory-specific proteins, namely, odorant-binding proteins (OBPs), odorant receptors (ORs), and odorant-degrading enzymes. The relationship between the pheromone structures and the primary sequences of the proteins suggest that OBPs play a part in the selectivity of the olfactory system in scarab beetles by "filtering" chemical signals during the early olfactory processing (perireceptor events). Nevertheless, it is unlikely that pheromone-binding proteins are "chiral filters" as the Japanese and Osaka beetles each possess only one single binding protein. Upon interaction with negatively charged membranes, OBPs undergo conformational changes that may lead to the release of the ligands.
\end{abstract}

\section{PHEROMONE CHEMISTRY}

Recent studies have led to the identification of the sex pheromones of various species in the subfamily Rutelinae and Melolonthinae [1]. In general, the pheromones of the former are fatty acid-derived compounds [2], whereas the latter utilizes phenolic, terpenoid, and amino acid-derived compounds. Two interesting exceptions to this general rule are the pheromones of Heptophylla picea and Phyllopertha diversa. Although belonging to the Melolonthinae, H. picea utilizes (R,Z)-7,15-hexadecadien-4-olide [3], most likely biosynthesized from stearic acid. On the other hand, P. diversa (Rutelinae) produces an intriguing alkaloid pheromone, which also has medicinal properties [4].

Utilizing pheromone blends that consist of just a few semiochemicals or even a single constituent, closely related species have attained isolated chemical communication channels and reproductive isolation $[5,6]$. Species that have the same pheromone system are isolated either temporarily or geographically. Interestingly, Anomala osakana and Popillia japonica utilize enantiomers of a chiral pheromone (japonilure), with one stereoisomer being an attractant and the other a behavioral antagonist. P. japonica and A. osakana produce (R)- and (S)-japonilure, respectively $[7,8]$. The pheromone of one species is a behavioral antagonist for the other. It seems that this agonist-anatagonist activities of the enantiomeric pheromones have evolved as part of the isolation mechanism between these two species that

*Lecture presented at the $22^{\text {nd }}$ IUPAC International Symposium on the Chemistry of Natural Products, São Carlos, Brazil, 3-8 September 2000. Other presentations are published in this issue, pp. 549-626. 
share the same habitats in Japan. In general, scarab beetles can detect only the enantiomer produced by the conspecific females, but $P$. japonica and A. osakana have evolved the ability to detect both enantiomers, one as an attractant and the other as a behavioral antagonist (stop signal).

\section{MOLECULAR BASIS OF OLFACTION}

For their survival, insects heavily depend on their ability to detect chemical signals from the environment, which are buried in complex mixture of odors from myriad sources. This has been highlighted in the literature by their highly sensitive and selective olfactory systems for the detection of sex pheromones, particularly in Lepidoptera, which approach the theoretical limit for a detector. While minimal structural modifications to pheromone molecules render them inactive [9], a single molecule of the native ligand is reported to be sufficient to activate the pheromone-specific olfactory neurons in the antennae of the silkworm moth, Bombyx mori [10]. There is growing evidence in the literature that this inordinate sensitivity is achieved by a combination of the roles of various olfactory specific proteins, including odorant receptors, odorant-binding proteins, and odorant-degrading enzymes. In order to gain a better understanding of the molecular basis of olfaction, we aimed at identifying and characterizing the pheromone-degrading enzymes, studying the neurophysiological details of pheromone perception in vivo, and isolating, identifying, and cloning the genes encoding the pheromone- and odorant-binding proteins. In order to elucidate the function(s) of these proteins, we have been conducting structural studies in collaboration with Jon Clardy (Cornell University) and Kurt Wuthrich (ETH-Switzerland).

\section{PHEROMONE-DEGRADING ENZYMES}

Antennal proteins from the extracts of several species of scarab beetles can degrade buibuilactone and japonilure, even those from species that do not use this group of compounds as their pheromones. In some cases there was only one metabolite, identified as the corresponding hydroxy fatty acid. It seems that the deactivation of the lactone signal is obtained by the opening of the lactone ring. Some species, however, degraded the pheromone into several more products. The esterase from A. octiescostata showed significant preference for $(\mathrm{R})$-japonilure over that of the $(\mathrm{S})$-enantiomer. This observation is consistent with the fact that this species produces only the $(\mathrm{R})$-enantiomers of the two pheromone components and it is anosmic to the (S)-lactones.

Analysis of the degradation products of the unique pheromone from $P$. diversa revealed that only the antennal extract of this species can degrade the pheromone. The antennal extracts from 10 other scarab species and 4 lepidoptearn species produced no activity at all. Separation of the antennal extracts showed that the enzymatic activity was associated with the membrane fractions in the absence of cytosol. Analysis of the degradation reaction suggested that the major degradation product was due to a demethylation at the $\mathrm{N}-1$ position; the second product was due to hydroxylation of the aromatic ring. Studies on the degradation along with potential cofactors or inhibitors showed that the enzymatic system requires NADPH and NADH for activity. On the other hand, the enzymatic activity was inhibited by proadifen and metyrapone, two general widely used inhibitors for cytochrome P450 [11].

\section{DEGRADATION OF PHEROMONES IN VIVO}

The discovery of the unique pheromone-degrading enzyme in $P$. diversa and the identification of enzymatic inhibitors opened the way to study pheromone inactivation in vivo. When metyrapone was introduced by diffusion into the pheromone-specific sensilla in the antennae of $P$. diversa, the pheromone detectors became "silent" to lower concentrations after application of a large concentration of the pheromone. The effect of the inhibitor is remarkably different from adaptation. In addition, metyrapone treatment had no effect on the sennsila of $P$. diversa tuned to (Z)-3-hexenyl acetate nor did it affect the 
pheromone-detecting systems in P. japonica, for which pheromone inactivation is achieved with a sensillar esterase.

\section{IDENTIFICATION AND CLONING OF OBPS}

We have identified, cloned, and characterized the odorant-binding proteins from a number of scarab species. It is now clear that scarab beetles possess two families of odorant-binding proteins, one with 116 and the other with 133 amino acids, which we named OBP1 and OBP2, respectively. While OBP1 is well conserved among all species of scarab beetles, OBP2 belongs to a more diverse group and, in contrast to OBP1, it has not been detected in all species. Interestingly, OBP2 possesses isoforms, which can be separated by native gel electrophoresis. These isoforms have different binding affinities. For example, one isoform of OBP2 from $P$. diversa binds bombykol, whereas the other conformation binds japonilure [12]. Microheterogeneity of the OBPs in scarab beetles is not derived from different gene products, but it is due to the conformational flexibility of the proteins. Consistently, we found only one gene encoding OBP2 in various species.

Interestingly, in both A. osakana and P. japonica, we could detect only one PBP in the antennal extracts; the proteins from the two species showed a $96 \%$ similarity. Due to the limited sensitivity of the detection methods, one cannot rule out the possibility of the presence of proteins expressed at low levels. However, electrophysiological experiments suggest that if two PBPs were involved in the signal transduction of the enantiomers of japonilure they would be expressed at nearly the same level. Single sensillum recordings from the antennae of the Japanese and Osaka beetles showed that enantiospecific receptor neurons respond equally to (R)- and (S)-japonilure. These findings and the observation that a single PBP from A. osakana bound both enantiomers of japonilure apparently with the same affinity suggested that in the antennae of these species, the same PBP may recognize both the pheromone and the "stop signal", i.e., the enantiomers of japonilure [13].

\section{STRUCTURAL BIOLOGY AND FUNCTION OF PBPS}

We envisaged that in order to determine the molecular basis of insect olfaction and elucidate the function of PBPs, we needed to study the 3D structure of the pheromone-binding proteins and its interaction with ligands. We embarked in collaborations with two groups (Jon Clardy and Kurt Wuthrich) to determine the 3D crystal and solution structures of the pheromone-binding protein from Bombyx mori. Functional expression of BmPBP was achieved in E. coli periplasm. The protein appeared as a single band in gel electrophoresis, and it was homgeneous in most chromatographic systems. However, NMR experiments conducted by the Wuthrich group indicated the existence of two conformations: an acidic form at $\mathrm{pH}$ below 4.9 and a basic form at $\mathrm{pH}$ above 6 [14]. Throughout the analysis of both the native and recombinant proteins, a remarkable feature of the PBPs appeared. These proteins have dynamic structures, altering their conformations in $\mathrm{pH}$-dependent ways. Studies with model membranes suggested that upon an interaction with the dendritic membrane, PBPs undergo a conformational change that may lead to the release of the pheromone ligand [15].

The 3D structure of the BmPBP with bound bombykol has been determined by X-ray diffraction [16]. BmPBP has six helices, and bombykol binds in a completely enclosed hydrophobic cavity formed by four antiparallel helices. Bomkykol is bound in this cavity through numerous hydrophobic interactions. It has been suggested that a $\mathrm{pH}$ drop would result in protonation of the histidine residues that form the base of a flexible loop and protonated histidines could destabilize the loop covering the binding pocket.

Although the crystal structure did not show clear evidence for dimers, a comprehensive study (Western immunoblotting experiments, mass spectral analysis, gel filtration estimation of molecular masses, and cross-linking reactions), showed that $\mathrm{BmPBP}$ is a monomer at acid $\mathrm{pH}$ and a dimer at basic, neutral, and slightly acid $\mathrm{pH}$. This suggests that the physiologically relevant $\mathrm{pH}$ for the early olfactory 
processing is not only that of the sensillar lymph (bulk $\mathrm{pH}$ ), but also the $\mathrm{pH}$ at the surface of the dendrides (localized pH) [17].

\section{ACKNOWLEDGMENTS}

I gratefully acknowledge the great contribution that my past and present collaborators and members of my research group made to this work. My research projects in Japan were financially supported by a special coordination fund for promoting science and technology by the Science and Technology Agency of Japan and by the Program for Promotion of Basic Research Activities for Innovative Biosciences (BRAIN). Work in the United States was made possible through direct financial support from the department, college, and Chancellors office at UCD.

\section{REFERENCES}

1. W. S. Leal. Annu. Rev. Entomol. 43, 39 (1998).

2. W. S. Leal, P. H. G. Zarbin, H. Wojtasek, J. T. Ferreira. Eur. J. Biochem. 259, 175 (1999).

3. W. S. Leal, S. Kuwahara, M. Ono, S. Kubota. Bioorg. Med. Chem. 4, 315 (1996).

4. W. S. Leal, P. H. G. Zarbin, H. Wojtasek, S. Kuwahara, M. Hasegawa, Y. Ueda. Nature 385, 213 (1997).

5. W. S. Leal. In Pheromones of Non-lepidopteran Insects Associated with Agricultural Plants, J. Hardie and A. K. Minks (Eds.), pp. 51-68. CABI Publishing, New York (1999).

6. W. S. Leal. In Mechanisms of Chemical Communication in Scarab Beetles, T. Hidaka, Y. Matsumoto, K. Honda, H. Honda, K. Tatsuki (Eds.), pp. 464-478, University of Tokyo Press, Tokyo (1999).

7. J. H. Tumlinson, M. Klein, R. E. Doolitle, A. T. Proveaux. Science 197, 789 (1977).

8. W. S. Leal. Proc. Natl. Acad. Sci. USA 93, 12112 (1996).

9. K. E. Kaissling. In R. H. Wright Lectures on Insect Olfaction, K. Colbow (Ed.), pp. 28-32, Simon Fraser University, Burnaby, British Columbia (1987).

10. K. E. Kaissling and E. Priesner. Naturwissenschaften 57, 23 (1970).

11. H. Wojtasek and W. S. Leal. FEBS Lett. 458, 333 (1999).

12. H. Wojtasek, J.-F. Picimbon, W. S. Leal. Biochem. Biophys. Res. Commun. 263, 832 (1999).

13. H. Wojtasek, B. S. Hansson, W. S. Leal. Biochem. Biophys. Res. Commun. 250, 217 (1998).

14. F. Damberger, L. Nikonova, R. Horst, G. Peng, W. S. Leal, K. Wuthrich. Protein Sci. 9, 1038 (2000).

15. H. Wojtasek and W. S. Leal. J. Biol. Chem. 274, 30950 (1999).

16. B. H. Sandler, L. Nikonova, W. S. Leal, J. Clardy. Chem. Biol. 7, 143 (2000).

17. W. S. Leal. Biochem. Biophy. Res. Commun. 268, 521 (2000). 